\title{
Electric Characterization and Modeling of Microfluidic-Based Dye-Sensitized Solar Cell
}

\author{
Adriano Sacco, ${ }^{1,2}$ Andrea Lamberti, ${ }^{1,2}$ Marzia Quaglio, ${ }^{1}$ Stefano Bianco, ${ }^{1}$ Elena Tresso,, 2 \\ Anca-Luiza Alexe-Ionescu, ${ }^{1,3}$ and Candido F. Pirri ${ }^{1,2}$ \\ ${ }^{1}$ Center for Space Human Robotics @Polito, Istituto Italiano di Tecnologia, Corso Trento 21, 10129 Torino, Italy \\ ${ }^{2}$ Dipartimento di Scienza Applicata e Tecnologia, Politecnico di Torino, Corso Duca degli Abruzzi 24, 10129 Torino, Italy \\ ${ }^{3}$ Faculty of Applied Sciences, University Politehnica of Bucharest, Splaiul Independentei 313, 060042 Bucharest, Romania
}

Correspondence should be addressed to Stefano Bianco, stefano.bianco@iit.it

Received 3 November 2011; Accepted 4 January 2012

Academic Editor: Latika Menon

Copyright (c) 2012 Adriano Sacco et al. This is an open access article distributed under the Creative Commons Attribution License, which permits unrestricted use, distribution, and reproduction in any medium, provided the original work is properly cited.

\begin{abstract}
The electric response to an external periodic voltage of small amplitude of dye-sensitized solar cells (DSCs) made up with an alternative architecture has been investigated. DSCs have been fabricated with a reversible sealing structure, based on microfluidic concepts, with a precise control on the geometric parameters of the active chamber. Cells with different electrolyte thicknesses have been characterized, without varying the thickness of the $\mathrm{TiO}_{2}$ layer, both under illumination and in dark conditions. Measurements of the electric impedance have been performed in the presence of an external bias ranging from $0 \mathrm{~V}$ to $0.8 \mathrm{~V}$. The experimental data have been analyzed in terms of a transmission line model, with two transport channels. The results show that the photovoltaic performances of the microfluidic cell are comparable with those obtained in irreversibly sealed structures, actually demonstrating the reliability of the proposed device.
\end{abstract}

\section{Introduction}

Since the first paper of O'Regan and Grätzel [1], dyesensitized solar cells (DSCs) have been widely investigated as one of the most promising candidates for the next-generation solar devices with low production costs, simple fabrication process, and good efficiency in energy conversion. It is easy to obtain DSC samples employing commercially available materials and simple process steps, nevertheless, the performances are strongly dependent on the material quality and the fabrication procedure.

Newly implemented manufacturing solutions such as advanced print screen techniques, electrolyte filling, dye profiling, and sealing machines have been reported for obtaining small laboratory DSC with efficiency up to $10 \%$, good stability, and reproducibility [2-6]. The present DSC research and development focus on finding materials and manufacturing techniques for higher conversion efficiency, lower costs, and longer operating lifetime. In this process, the understanding of the influence of materials and components on the overall efficiency is essential. Experimental measurements provide a basis for identifying the factors mostly limiting the efficiency of a DSC. Furthermore, when coupled to mathematical models, they can provide a quantitative understanding of the device's physics [7]. Also, numerous papers have been published on modeling and interpreting the results obtained from electrochemical impedance spectroscopy (EIS) measurements [7-16].

Several papers have been published suggesting the best fabrication procedure of the cells $[2,17,18]$ and, moreover, in the papers reporting experimentalresults, the procedure used to obtain small laboratory cells is quite always illustrated in details. Even if sometimes a simple-clips closure has been chosen, the use of hot-melt sealants is generally adopted for closure and protection from the environment. For research purposes, this can be somewhat limiting. Indeed, some of the fabrication steps, as dye adsorption and electrolyte filling, are often performed without any direct/active control, being difficult to ensure reproducibility and reliability while fabricating a large number of cells. Both during experiments and after them, the cell, being irreversibly sealed, does not 
grant the possibility of control and inspection, or postprocess modifications.

Recently, our group has proposed a new technological procedure for DSC fabrication [19], which is quite familiar to the field of microfluidics. We designed a cell that is actually made by all the parts of the traditional Grätzel's device, in which a microfluidic chamber has been designed by means of a polydimethylsiloxane (PDMS) membrane. PDMS exhibits a good spontaneous reversible adhesion to glass, metals, and oxides. The membrane acts as a spacer between the two electrodes, defining the inner active volume of the DSC. To ensure a good sealing, the $\mathrm{TiO}_{2}$ layer is designed having a circular shape entirely confined into the microfluidic chamber. This is obtained performing a casting step into a tape-mould having a hole with a fixed diameter into it. To definitively close the device, a housing system has been designed, with an external clamp closed by screws. In this way, the sealing procedure is reversible, granting inspection and control also after the experiments. Sealing performances of such innovative structure were successfully evaluated by dynamic fluidic tests. Our aim was the engineering of a small laboratory cell which permits to distinguish between the contributions of the different components and technological steps. We employed a microfluidic device which offers good flexibility and still grants low-cost materials and technologies together with the use of very small amounts of reagents. Our modular device enables to substitute one or more components and guarantees the reproducibility of the assembly parameters such as load distribution, screw tightening, and thicknesses control. We have devoted particular care in the definition of the active surface, in the control of thickness uniformity and of the dye impregnation.

In this paper, commercially available DSC materials have been used to fabricate a set of microfluidic solar cells, with two different electrolyte thicknesses. Electric characterization has been performed through standard $I-$ $V$ measurements and through EIS measurements under light and dark conditions, at different bias voltages. The experimental data are analyzed in terms of a transmission line model, with two transport channels, to validate already proposed models [20]. This validation is fundamental since it shows that our microfluidic approach does not change the nature of the device but simply adds flexibility and reliability. We demonstrate the effectiveness of the microfluidic cells as a standard modular prototype which can be used for testing different DSC components.

The paper is organized as follows. In Section 2, we describe the microfluidic cell preparation procedure. In Section 3, the transmission line model which is used to fit the experimental data is briefly recalled. In Section 4, experimental results are presented and the best fit parameters of our data are discussed. Section 5 is devoted to the conclusions.

\section{Experimental}

Fluorine-doped-tin-oxide- (FTO) covered glasses $(7 \Omega / s q$, Solaronix) were rinsed with acetone and ethanol in an ultrasonic bath for $10 \mathrm{~min}$. Then, a $\mathrm{TiO}_{2}$ layer (Ti-Nanoxide D37 paste, Solaronix) with a circular shape (with a fixed diameter of $(10.00 \pm 0.05) \mathrm{mm})$ was deposited on FTO by tape-casting technique and dried at $50^{\circ} \mathrm{C}$ for $30 \mathrm{~min}$ on a hot plate. A sintering process at $450^{\circ} \mathrm{C}$ for $30 \mathrm{~min}$ allowed the formation of nanoporous $\mathrm{TiO}_{2}$ film with a mean thickness of $(7.5 \pm 0.5) \mu \mathrm{m}$, as measured by profilometry (P.10 KLATencor).

Photoelectrodes were soaked into a $0.2 \mathrm{mM}$ N719 dye solution (Ruthenizer535bis-TBA, Solaronix) in ethanol for $24 \mathrm{~h}$ at room temperature and then rinsed in pure ethanol to remove the unadsorbed molecules. Two small pin holes for inlet/outlet connections were drilled in the FTO glass counter electrodes through powder-blasting technique. Substrates were then cleaned with the same rinsing method described above and a $5 \mathrm{~nm}$ Pt layer was deposited onto FTO by thermal evaporation. The inlet/outlet ports for electrolyte (Iodolyte AN 50, Solaronix) filling were connected via low-density polyethylene tubes and closed for operation with PDMS caps.

The operating chamber was defined through a PDMS membrane, prepared by casting technique. PDMS prepolymer and curing agent (Sylgard 184, Dow Corning) were mixed in a $10: 1$ weight ratio and degassed in low vacuum for $1 \mathrm{~h}$. The mixture was then poured into the mould and cured in a convection oven for $1 \mathrm{~h}$ at $70^{\circ} \mathrm{C}$. The membrane was then peeled off from the mould and reversibly sandwiched between the electrodes. Accurate control in the volume of the mould and on PDMS weight during casting process allowed a precise control in membrane thickness and uniformity. The PDMS membrane laterally defines the active chamber of the cell. For our microfluidic cells, two different membrane thicknesses have been chosen, $(200 \pm 15) \mu \mathrm{m}$ and $(100 \pm$ 8) $\mu \mathrm{m}$, for obtaining one "thick" and one "thin" chamber, respectively. A double-drop membrane layout was chosen to promote air bubble evacuation during electrolyte filling. The device is closed by an external housing system (made of two poly(methyl methacrylate) frames) that clamps the two electrodes and allows fluids handling. A $50 \mu \mathrm{m}$ retaining ring is designed on the membrane to follow the profile of the chamber ensuring sealing by completely deforming when closing the device with screws. The complete deformation of the retaining ring grants the final distance between the electrodes to coincide with the thickness of the membranes. The strength applied to the screws is controlled by a torque spanner. In this way, the sealing procedure is reversible, allowing inspection and control after the experiments.

All cells had an active area of $0.78 \mathrm{~cm}^{2}$ and measurements were performed with a $0.22 \mathrm{~cm}^{2}$ shadow mask. Copper foils $\left(50 \mu \mathrm{m}\right.$ thick, area $\left.1.5 \mathrm{~cm}^{2}\right)$ were used for electric connections at the electrodes, dielectrically isolated by the PDMS membrane.

Impedance analysis was done using a potentiostat $(760 \mathrm{D}$, $\mathrm{CH}$ Instruments) in a two-electrode configuration: reference and counter electrodes were linked together to DSC cathode, while working electrode was connected to the cell photoanode. The amplitude of the AC signal was $10 \mathrm{mV}$, and the frequency was varied in the range $10^{-1}-10^{4} \mathrm{~Hz}$. 
The applied bias potential has been varied in the range 0 $0.8 \mathrm{~V}$. Measurements were taken both under 1 sun $\mathrm{AM} 1.5 \mathrm{G}$ illumination, using a class A solar simulator (91195A, Newport), or in dark conditions.

\section{Model}

In electrochemical cells, the transport and charge-transfer processes are described by nonlinear equations connecting the driving forces to the flux of the particles. The impedance spectroscopy technique is based on the analysis of the electric response of a cell to a periodic external electric excitation of variable frequency. When the amplitude of the applied voltage is comparable with the thermal voltage, $V_{\text {th }}=$ $k_{B} T / q$, the fundamental equations describing the charge redistribution in the cell due to the external voltage are linear [21]. In this approximation, it is possible to describe all the processes by linear equations in which the coefficient between the electrochemical potential and the current is the electric impedance. In this framework, the electrochemical cell can be described by an equivalent electric circuit.

The performances of the DSC are connected with the morphology and structure of the $\mathrm{TiO}_{2}$ layer on which the dye molecules are attached, with the ability of the electrolyte of regenerating the photoabsorbing molecules and also with the contact between the solid film or the electrolyte and the conductive electrodes. All these processes have characteristic times ranging from hundreds of seconds to microseconds, which, transposed into the frequency domain, correspond to the $\mathrm{mHz}$ to $\mathrm{MHz}$ part of the electromagnetic spectrum. The impedance spectroscopy technique allows separating different processes occurring in such very complex systems. From the impedance spectra, it is possible to analyze the diffusion-recombination electronic processes in the $\mathrm{TiO}_{2}$ film and at the interface solid film-electrolyte, the diffusion mechanism of the redox species in the electrolyte, and also the charge transfer at the electrodes. The diffusion time of the redox species strongly depends on the thickness of the electrolyte and this, in turn, is connected to the overall thickness of the cell.

A porous film in contact with an electrolyte is described by a transmission line model with two transport channels and crosswise elements [20]. The carrier transport is simultaneously of electronic origin in the solid phase and ionic in the liquid. Both media in contact are supposed to be homogenous and continuously connected phases. Due to the special geometrical structure of the complex system film-electrolyte, there will be a distribution of the electric current generated by the charges moving along each media and also on the direction normal to the internal surface. The total electric current can be expressed as a sum of AC currents, $i_{1}$ and $i_{2}$, flowing in the liquid and solid phase, respectively. The impedance of the solid film in contact with the electrolyte is obtained by solving the equations for the electric currents and their local variations in both phases $[22,23]$. The charge transport in the solid or liquid phases is simply showed by resistances. The electrolyte-solid film interface and the associated electrochemical processes are described as an electric circuit formed by a parallel of a capacitor and a resistance:

$$
Z_{R}=\frac{R_{\mathrm{tr}} \cdot R_{e}}{R_{\mathrm{tr}}+R_{e}}\left(1+\frac{2 \lambda / d}{\sinh (d / \lambda)}\right)+\frac{\lambda}{d} \frac{R_{\mathrm{tr}}^{2}+R_{e}^{2}}{R_{\mathrm{tr}}+R_{e}} \operatorname{coth}\left(\frac{d}{\lambda}\right),
$$

where

$$
\frac{\lambda}{d}=\sqrt{\frac{Z_{c t}}{R_{\mathrm{tr}}+R_{e}}} .
$$

In (1), $R_{\mathrm{tr}}$ and $R_{e}$ are the transport resistance in the solid film and the diffusion resistance in the electrolyte, respectively, and $d$ is the thickness of the porous film [20]. Equation (1) has been obtained by imposing that the ionic current is zero at the end of the pore in the film and also that the electronic current in the solid film is zero at the outer edge of the film. These suppositions are assumed for the situation in which the edges of the solid film are planar and perfectly reflecting.

In (2), $Z_{\mathrm{ct}}$ is the equivalent impedance formed by a parallel $R_{\mathrm{ct}}$ and $C_{t}$ :

$$
Z_{\mathrm{ct}}=\frac{1}{i \omega C_{t}+R_{\mathrm{ct}}^{-1}}
$$

where $C_{t}$ is the total capacitance in the solid film and within the interface, $R_{\mathrm{ct}}$ is the charge-transfer resistance at the solid-electrolyte interface. This description is related to the presence of both faradaic and polarization currents in the region separating the two phases. In terms of resistance and capacitance connected to the solid-electrolyte interface, it is possible to define a characteristic time $\tau_{n}=\left(\omega_{n}\right)^{-1}=R_{\mathrm{ct}} C_{t}$ associated to the electron lifetime in the solid film.

The collection efficiency of the charges at the front electrode requires that the electron diffusion length, $L_{n}$, is greater than the thickness of the porous film. The electron diffusion length is related to the ratio between the charge transport time, $\tau_{\mathrm{tr}}=R_{\mathrm{tr}} C_{t}$ and the charge lifetime, $\tau_{n}=$ $R_{\mathrm{ct}} C_{t}, L_{n}=d\left(\tau_{n} / \tau_{\mathrm{tr}}\right)^{1 / 2}$ [23]. For good charge collection efficiency, the charge-transfer resistance has to be larger than the transport resistance.

The impedance describing the diffusion of the redox species in the electrolyte has the form:

$$
Z_{e}=R_{e} \frac{\tanh \left(\sqrt{\left(i \omega / \omega_{d}\right)}\right)}{\sqrt{\left(i \omega / \omega_{d}\right)}}
$$

where $\omega_{d}$ is the characteristic frequency of the diffusion [24].

The contact between the electrolyte and the counter electrode is modeled by a parallel $R_{p} C_{p}$, with the equivalent impedance:

$$
Z_{p}=\frac{1}{i \omega C_{p}+R_{p}^{-1}},
$$

where $C_{p}$ is the interfacial Helmholtz (double-layer) capacitance and $R_{p}$ is the charge-transfer resistance at the counter electrode [25].

It follows that the total impedance for a DSC is

$$
Z=R_{s}+Z_{R}+Z_{e}+Z_{p},
$$


where $R_{s}$ is the resistance of the leads and of the ohmic contacts to the cell.

The different contributions to the total impedance $Z$ of the cell depend on the frequency in different manners. From the fit of the full spectra of its real and imaginary parts, we can derive information on the different mechanisms responsible for the conduction across the DSC.

The best fit of the experimental data is obtained by minimizing the quantity:

$s=\frac{\sum_{i=n_{i}}^{n_{f}}\left|R\left(\omega_{i}\right)-R_{\exp }\left(\omega_{i}\right)\right|}{2\left(n_{f}-n_{i}+1\right) \bar{R}_{\exp }}+\frac{\sum_{i=n_{i}}^{n_{f}}\left|X\left(\omega_{i}\right)-X_{\exp }\left(\omega_{i}\right)\right|}{2\left(n_{f}-n_{i}+1\right) \bar{X}_{\exp }}$,

where $R\left(\omega_{i}\right)=\operatorname{Re} Z\left(\omega_{i}\right)$ is the real part of the total impedance, $R_{\exp }\left(\omega_{i}\right)$ is the measured value, $\bar{R}_{\exp }$ is the average of the experimental values $R_{\exp }\left(\omega_{i}\right)$, and $\left(n_{f}-n_{i}+1\right)$ is the total number of experimental points. Similar definitions stand for the imaginary part of the impedance $X=\operatorname{Im}(Z)$. The factor 2 in the denominator was used to make $s=1$ if $Z(\omega) \equiv 0$. We have chosen to express the minimizing function $s$ in terms of absolute values of $R\left(\omega_{i}\right)-R_{\exp }\left(\omega_{i}\right)$ and not, as usual, in terms of squares of these deviations, for the reason that the minimizing procedure was entirely numerical, no derivatives were necessary and, numerically, the evaluation of the absolute value is less time consuming than squaring.

\section{Results and Discussion}

4.1. Efficiency and Reproducibility of the Microfluidic Solar Cell. The microfluidic solar cells have been designed to permit control, reproducibility, and reliability in a simple system. Moreover, the reversibility of the sealing is designed to allow the inspection of the prototypes after usage. A significant attention has been devoted to the characterization of the reproducibility of the photovoltaic conversion efficiency. In Figure 1(a) we report a scheme of a microfluidic DSC in which all the elements constituting the cell are exploded, in Figure 1(b) a picture of the final device, and in Figure 1(c) the evaluation of the sun energy conversion efficiency for a statistically significant number of identical cells. The evaluated conversion efficiency is $(6.76 \pm 0.11) \%$. The variation of the predicted efficiency value around the average value is comparable with the one obtained with irreversibly-sealed structure [2]. In Table 1, the obtained electric parameters are reported for typical "thin" and "thick" cells.

4.2. EIS Measurements. Several papers have reported on the impedance spectroscopy of DSC. However, only a few have investigated the electric response of the cells with different thicknesses of electrolyte, since standard cells do not allow modifying this parameter in a controllable manner. In what follows, we compare the Nyquist plots for DSC varying their thicknesses. Special attention is devoted to the electric response under light and dark conditions. In all reported Nyquist diagrams, we subtract the resistance of the electrode, responsible for the value of the impedance of the full system in the high-frequency limit. In this manner, the diagrams are relevant only to the electric properties of the cell.

In Figure 2, we compare the Nyquist diagrams corresponding to cells with different thicknesses $\left(L_{1}=200 \mu \mathrm{m}\right.$, $\left.L_{2}=100 \mu \mathrm{m}\right)$ subjected to bias voltages ranging from $0 \mathrm{~V}$ to $0.8 \mathrm{~V}$, under light (Figures $2(\mathrm{a})$ and $2(\mathrm{~b})$ ) and dark (Figures $2(\mathrm{c})$ and $2(\mathrm{~d})$ ) conditions. As it follows from Figure 2, in the high-frequency region the parametric plot of $-X$ versus $R$ is a straight line with a slope quite equal to 1 , and this result is independent of the thickness of the cell. The experimental results are in agreement with the model proposed by Bisquert [20] according to which in this frequency region the impedance of the DSC is mainly due to the electrolyte. In fact, for large frequency, $Z \rightarrow Z_{e}$.

By taking into account (4) and that in this region $\omega \gg$ $\omega_{d}$, we get

$$
Z \longrightarrow Z_{e} \approx R_{e} \sqrt{\frac{\omega_{d}}{2 \omega}}(1-i) .
$$

Consequently, the real and imaginary parts of $Z$ tend to

$$
\begin{gathered}
R \longrightarrow R_{e} \sqrt{\frac{\omega_{d}}{2 \omega}}, \\
-X \longrightarrow R_{e} \sqrt{\frac{\omega_{d}}{2 \omega}},
\end{gathered}
$$

respectively, and $-X=R$, indicating that the parametric plot of $-X$ versus $R$ is a straight line with the slope equal to 1 . A simple analysis shows that

$$
R_{e}=\frac{\alpha}{q D} \frac{L}{S},
$$

where $\alpha$ depends on the exchange of the charges at the electrode due to the adsorption, $q$ is the electric charge of the ion, $S$ is the effective surface of the porous electrode, and $D$ is the ionic diffusion coefficient.

Furthermore, the diffusion circular frequency is $\omega_{d}=$ $D / L^{2}$. Consequently, in the high frequency region,

$$
R \rightarrow R_{e} \sqrt{\frac{\omega_{d}}{2 \omega}}=\frac{\alpha}{q \sqrt{D}} \frac{1}{S} \sqrt{\frac{1}{2 \omega}} .
$$

This result indicates that both, in the considered frequency range, $R$ and $-X$ are independent of the thickness of the electrolyte. For the dark state, the behavior in the highfrequency range coincides with that in the light state.

In Figures 2(b) and 2(d), for the bias voltage of $0.6 \mathrm{~V}$ and the thickness of $L_{2}$, the different contributions to the impedance are not separable on the parametric plot. For larger bias voltages and thicknesses, the processes related to the counter electrode and $\mathrm{TiO}_{2}$ are well separated. It can be seen that the amplitude and the position of the peak corresponding to the charge transfer at the counter electrode are the same, for the same thickness and bias voltage, under light and dark conditions. On the contrary, the peak connected with the recombination in the $\mathrm{TiO}_{2}$ layer has smaller amplitudes for the light conditions and different positions. 


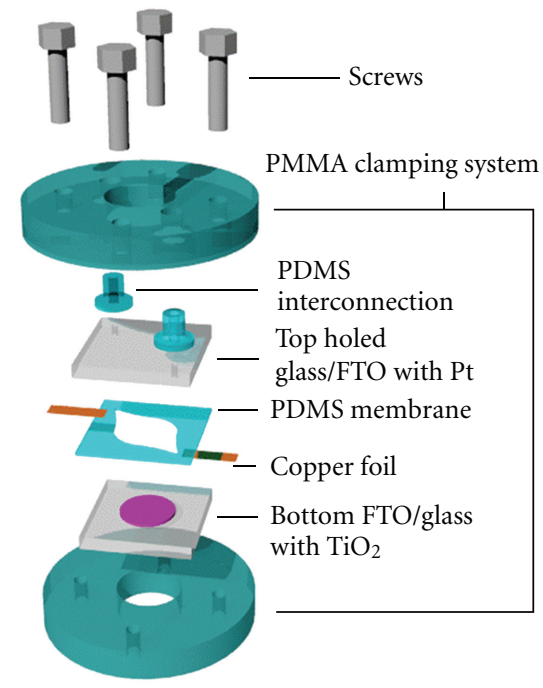

(a)

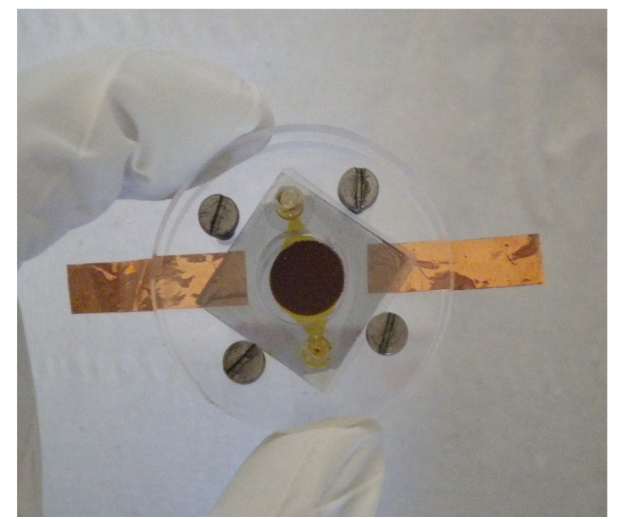

(b)

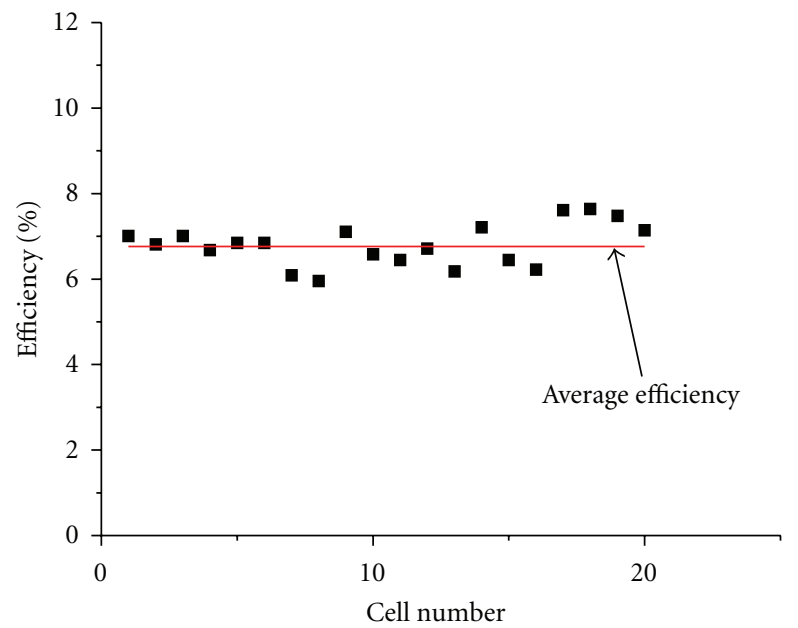

(c)

Figure 1: (a) Scheme of the microfluidic DSC with the detail of all the elements constituting the cell. (b) A picture of the final device. (c) Evaluation of the energy conversion efficiency for 20 nominally identical cells. Cell thickness was fixed at $200 \mu \mathrm{m}$.

TABLE 1: Electric parameters of two typical microfluidic cells with different thicknesses: $J_{S C}$, short circuit density of current, $V_{\mathrm{OC}}$, open circuit voltage, FF, fill factor.

\begin{tabular}{lcc}
\hline & $L_{1}=100 \mu \mathrm{m}$ & $L_{2}=200 \mu \mathrm{m}$ \\
\hline$J_{\mathrm{SC}}\left[\mathrm{mA} / \mathrm{cm}^{2}\right]$ & 19.5 & 20.5 \\
$V_{\mathrm{OC}}[\mathrm{V}]$ & 0.64 & 0.64 \\
$\mathrm{FF}$ & 0.58 & 0.55 \\
Efficiency $[\%]$ & 7.22 & 7.29 \\
\hline
\end{tabular}

In Figure 3(a), for the cell with $L_{1}=200 \mu \mathrm{m}$, we compare the Nyquist diagrams for three different bias voltages $(0.4 \mathrm{~V}$, $0.6 \mathrm{~V}$, and $0.8 \mathrm{~V}$ ) under illumination. For biases larger than $0.4 \mathrm{~V}$, the Nyquist diagram indicates the presence of two welldefined dissipative processes. The semicircles in the highfrequency limit, related to the counter electrode, have a radius which is a decreasing function of the bias voltage. The same conclusion holds true for the semicircle connected to $\mathrm{TiO}_{2}$ electrode appearing for moderate frequency. On the contrary, with our experimental accuracy, no conclusion can be derived for the semicircle related to the diffusion process in the electrolyte, appearing in the DC limit: the $0.1 \mathrm{~Hz}$ lower limit in EIS is not low enough for the cells, considering the redox ion used. Figure 3(b) refers to the same cell, under dark conditions. For large frequencies, the parametric plots of $-X$ versus $R$ are independent of the bias, and all the curves coincide, as evidenced in the zoom reported in Figure 3(b-1).

In Figure 4, the Nyquist diagrams for the two cells under the bias voltage of $0.4 \mathrm{~V}$ are compared: in Figure 4(a) under light and in Figure 4(b) under dark conditions. An interesting feature of the diagrams is that under illumination the resistance of the cell is larger for $100 \mu \mathrm{m}$ than for $200 \mu \mathrm{m}$, whereas for dark measurements the trend is reversed. The 


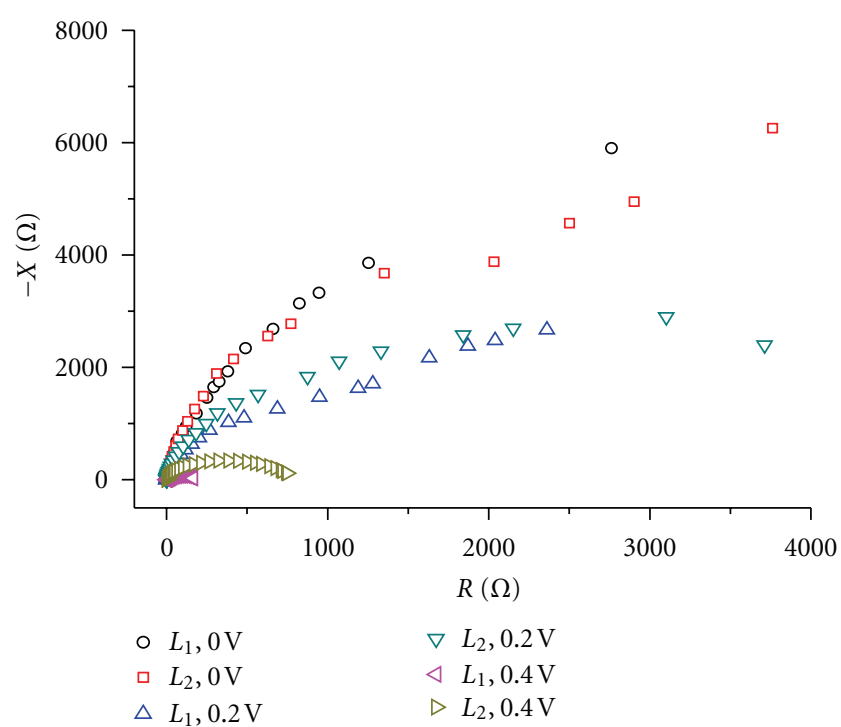

(a)

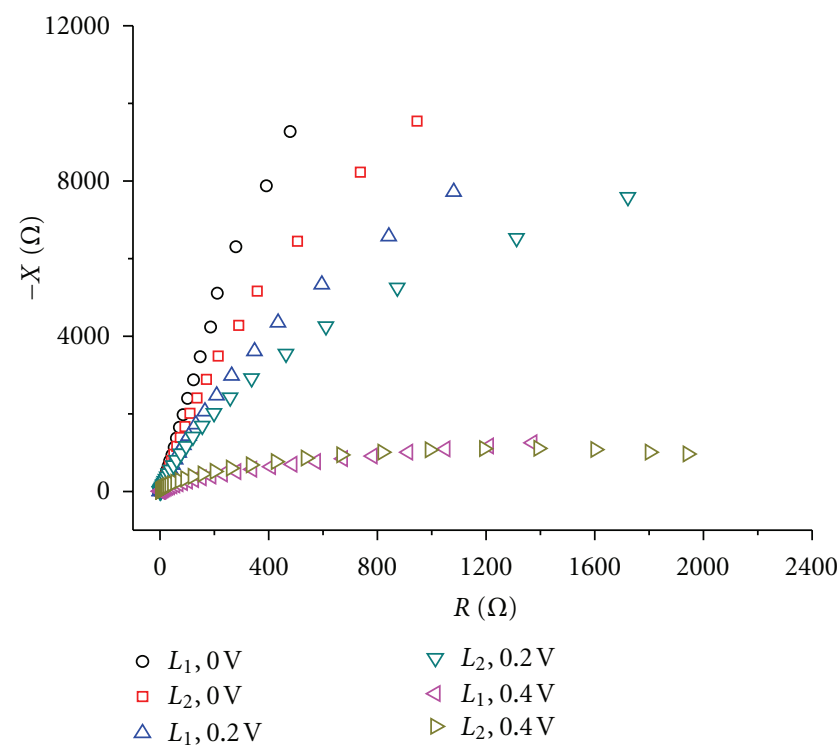

(c)

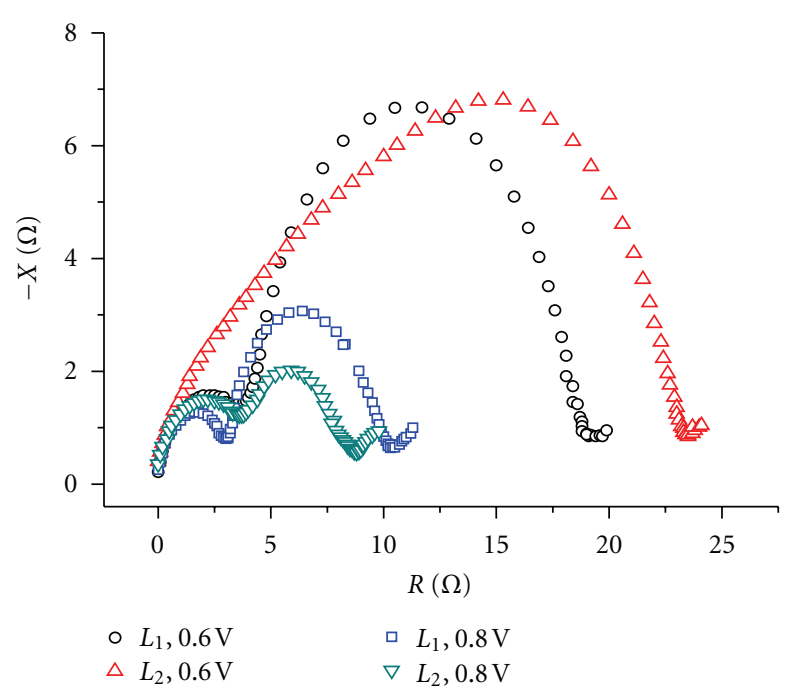

(b)

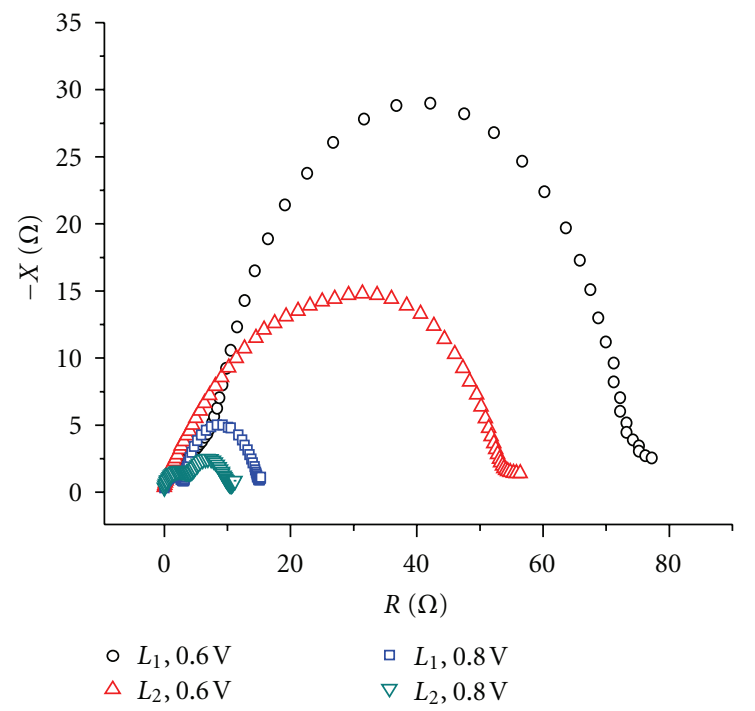

(d)

FIGURE 2: Nyquist diagrams for DSC with thicknesses $L_{1}=200 \mu \mathrm{m}$ and $L_{2}=100 \mu \mathrm{m}$, under bias voltages ranging from $0 \mathrm{~V}$ to $0.8 \mathrm{~V}$, under light $(\mathrm{a}, \mathrm{b})$ and dark (c, d) conditions.

same feature may be seen in Figure 5 for $0.6 \mathrm{~V}$ bias, but not for $0.8 \mathrm{~V}$ bias. These experimental results can be interpreted in terms of the following consideration. The effect of the electrolyte in the system is to furnish the ionic charges to regenerate the dye molecules adsorbed on the porous electrode. In the ideal case, each dye molecule would have an ion nearby in order to be immediately regenerated. For imaging a dynamic process in which the ions are positioned in front of the adsorbed layer of dyes in the pores, in the "thin" cell, for low biases $(\leq 0.6 \mathrm{~V})$, even if the liquid electrolyte is filling all the pores, it might happen that the ions within the pore are not enough to regenerate all the nearby molecules of the dye. In the thicker cell, the overall number of ions is larger, and so, although the equilibrium concentration is the same for the same bias, the number of ionic charges per unit surface within the pores, can be larger. For higher biases within all the pores, the dye molecules can be saturated by the ions even in thinner cells and the apparent resistance is given mainly by the resistance of the ionic film, the larger the film, the larger the resistance. Of course, for dark conditions, the regeneration process does not appear and the cells, behave normally, that is, thicker cells, larger resistances.

Until now, we just discussed the parametric plots showing $-X$ versus $R$, when the parameter is the frequency of the applied voltage. Some information can also be derived from the spectra of $R$ and $-X$. In this case, the presence of the different dissipative phenomena is indicated by plateau and 


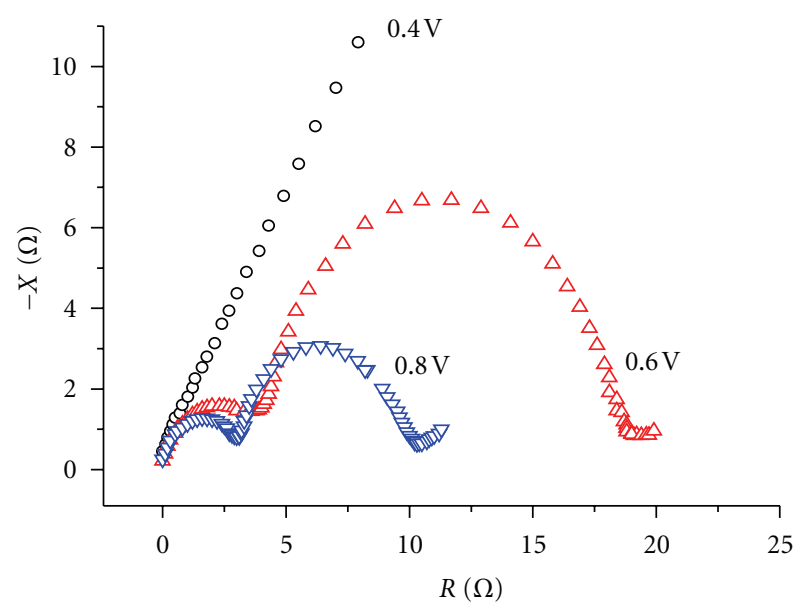

(a)

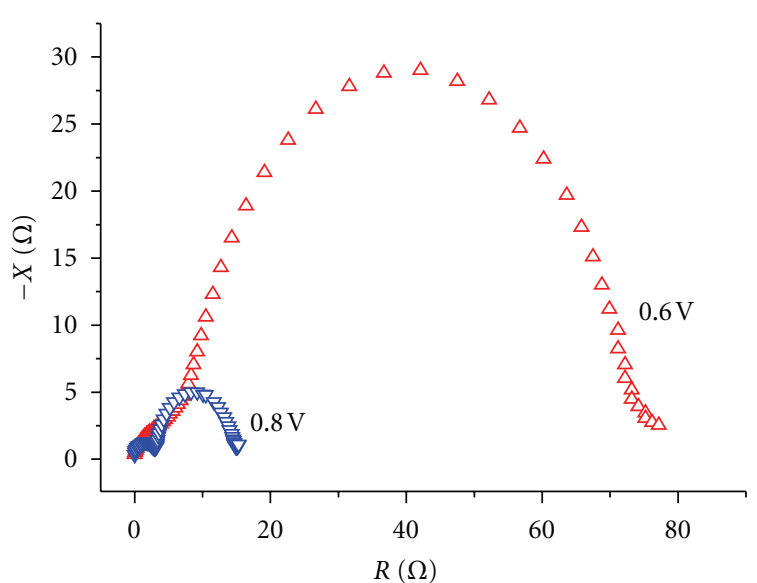

(b)

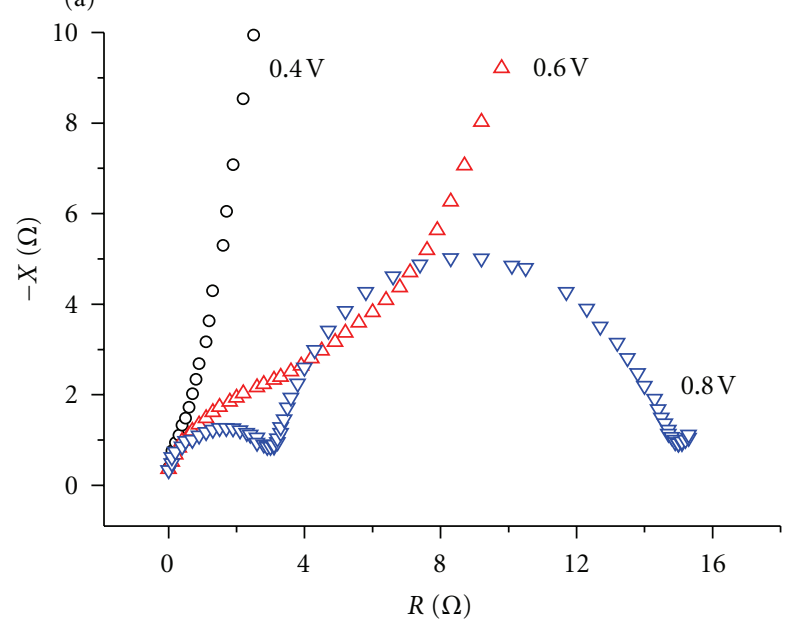

(b-1)

Figure 3: Nyquist diagrams for three different bias voltages $\left(0.4 \mathrm{~V}, 0.6 \mathrm{~V}\right.$ and $0.8 \mathrm{~V}$ ) corresponding to the cell of $L_{1}=200 \mu \mathrm{m}$, under light (a) and dark conditions (b). Zoom in the high-frequency region in and dark state (b-1).

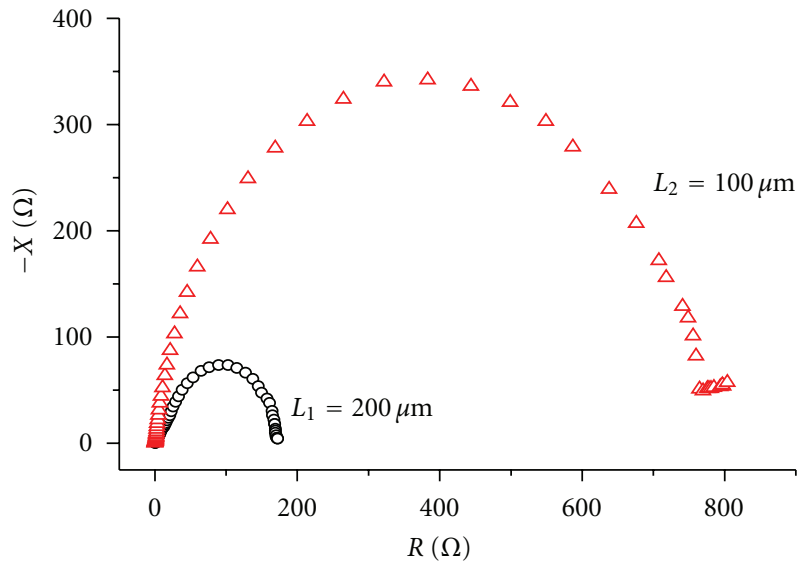

(a)

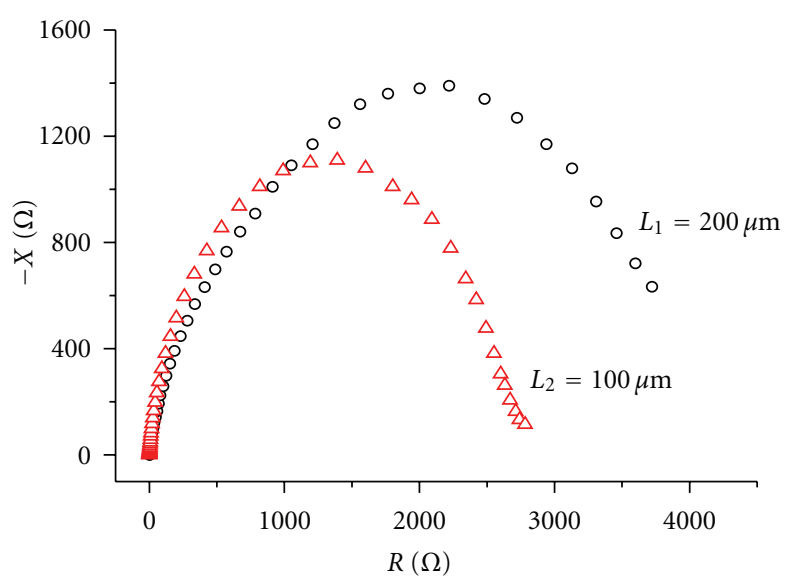

(b)

Figure 4: Nyquist diagrams for the cells with the thicknesses $L_{1}=200 \mu \mathrm{m}$ and $L_{2}=100 \mu \mathrm{m}$, under the bias voltage of $0.4 \mathrm{~V}$, in light (a) and dark (b) conditions. Note the different relative positions of the corresponding curves in the light and dark conditions. 


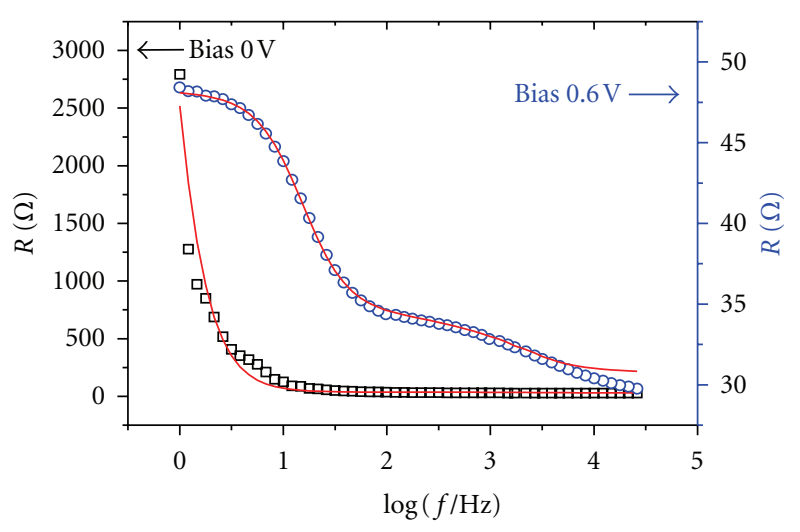

(a)

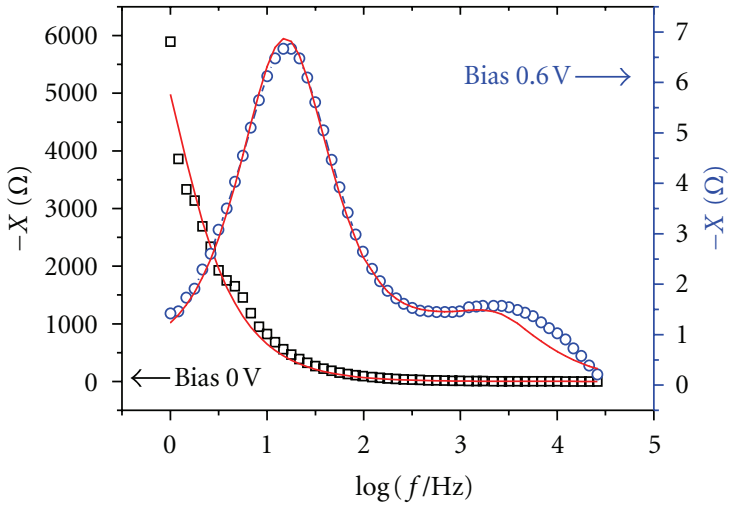

(b)

FIGURE 5: Real (a) and imaginary (b) parts of the impedance for the DSC with $L_{1}=200 \mu \mathrm{m}$ under illumination. Black squares and blue circles are the experimental data for bias of $0 \mathrm{~V}$ and $0.6 \mathrm{~V}$, respectively. Black and blue continuous curves are the corresponding best fits, obtained by means of the model described in the text.

maxima of $R$ and $-X$, respectively. The spectra of the real $(R)$ and imaginary $(-X)$ parts of the total impedance $Z$ of the cell given by (6) under light conditions are shown in Figures 5(a) and $5(\mathrm{~b})$, respectively. In these figures, the squares refer to the case in the absence of bias and the circles to a bias voltage of $0.6 \mathrm{~V}$.

As expected, the presence of the bias strongly reduces the resistance and the modulus of the reactance because the number of carriers increases. From Figure 5, we deduce that in the absence of any bias voltage, $R$ and $-X$ are monotonic functions of frequency (in the range between $1 \mathrm{~Hz}$ and $40 \mathrm{kHz}$ ). On the contrary, in the presence of bias voltage, the spectrum of $R$ presents two plateaux, one in the DC limit and the second one around $100 \mathrm{~Hz}$, whereas the spectrum of $-X$ presents a dissipation peak around $30 \mathrm{~Hz}$ related to the plateaux in the DC limit. The second peak is not visible in this limited range. Similar diagrams, not reported, can be derived in the experimental situation described in the previous figures. The best fit parameters of these curves, used in the characterization of $\mathrm{TiO}_{2}$-electrolyte interface, are discussed in the following section as far as their bias voltage dependence is concerned.

4.3. Characterization of the $\mathrm{TiO}_{2}$-Electrolyte Interface. To investigate the dependence of the photoanode-electrolyte interface impedance on the bias voltage, we performed measurements of the same kind reported in Figure 5 for bias voltages ranging from $0 \mathrm{~V}$ to $0.8 \mathrm{~V}$. The parameters of the best fit $R_{\mathrm{ct}}$ and $C_{t}$, characterizing this interface, versus the bias voltage $\mathrm{V}$, are shown in Figures 6(a) and 6(b), for the cells under light conditions. The triangles and stars refer to cells of thicknesses $100 \mu \mathrm{m}$ and $200 \mu \mathrm{m}$, respectively. We note that these quantities weakly depend on the thickness of the DSC, as expected, as they have to describe interface properties. The bias voltage dependence of $R_{\mathrm{ct}}$ and $C_{t}$ is well described by the model of Bisquert [20] according to which

$$
\begin{aligned}
R_{\mathrm{ct}} & =R_{0} \exp \left(-\beta V / V_{\mathrm{th}}\right), \\
C_{t} & =C_{0} \exp \left(\alpha V / V_{\mathrm{th}}\right),
\end{aligned}
$$

where $R_{0}$ and $C_{0}$ are the resistance and capacitance of the interface at $0 \mathrm{~V}$ bias, and $\beta$ and $\alpha$ are two constants $[22,25]$. From the best fit of Figure $6(\mathrm{a})$ and $6(\mathrm{~b})$, we find $\beta(100 \mu \mathrm{m})=0.13, \beta(200 \mu \mathrm{m})=0.17$, and $\alpha(100 \mu \mathrm{m})=0.15$, $\alpha(200 \mu \mathrm{m})=0.11$, values comparable with those reported in [20].

The difference between $\alpha$ and $\beta$ may be explained by the fact that $R_{\mathrm{ct}}$ depends on the free contact surface between the titania and the electrolyte (i.e., the total surface of the pore minus the surface occupied by the adsorbed dye molecules) whereas $C_{t}$ depends on the total surface of the pore (considering that the adsorbed dye molecular film minimally alters the dielectric constant in the interface).

$C_{t}$ is mainly related with the chemical capacitance that is connected to the carrier concentration in the conductive band or with the DOS at the $E_{c}$ position, but it depends also on surface interactions on $\mathrm{TiO}_{2}$ nanoparticles. In fact, a contribution to the capacitance has a dielectric origin and is related with the depletion at semiconductor/TCO or semiconductor/electrolyte interfaces, depending on voltage as $1 / \sqrt{V}[20,22]$. Moreover, one has to consider the double-layer capacitance in the electrolyte part of the semiconductor/electrolyte interface (in the case of concentrated electrolytes, it is known as Helmholtz capacitance). Thus, the dependence of $C_{t}$ on the bias voltage may not be a pure increasing exponential. The above formulae may be compared to the bias dependence of the carrier concentration in a semiconductor, namely, $n=n_{0} \exp \left(q V / k_{B} T\right)$, introducing an effective temperature $T_{\text {eff }}=T / \alpha($ or $T / \beta)$. As $T_{\text {eff }}>T$, we may say that to the entropic disorder characterized by $T$, in a single crystal semiconductor there is a supplementary disorder introduced by the porosity of titania and the adsorption of dye molecules.

At least, a part of $\alpha$ may correspond to a larger effective temperature in the case of porous media.

By means of the measured $R_{\mathrm{ct}}$ and $C_{t}$, we evaluate the relaxation time related to the charge transfer by means of $\tau_{n}=R_{\mathrm{ct}} C_{t}$, reported in Figure 6(c). From this figure, it follows that $\tau_{n}$ is practically independent on the thickness of 


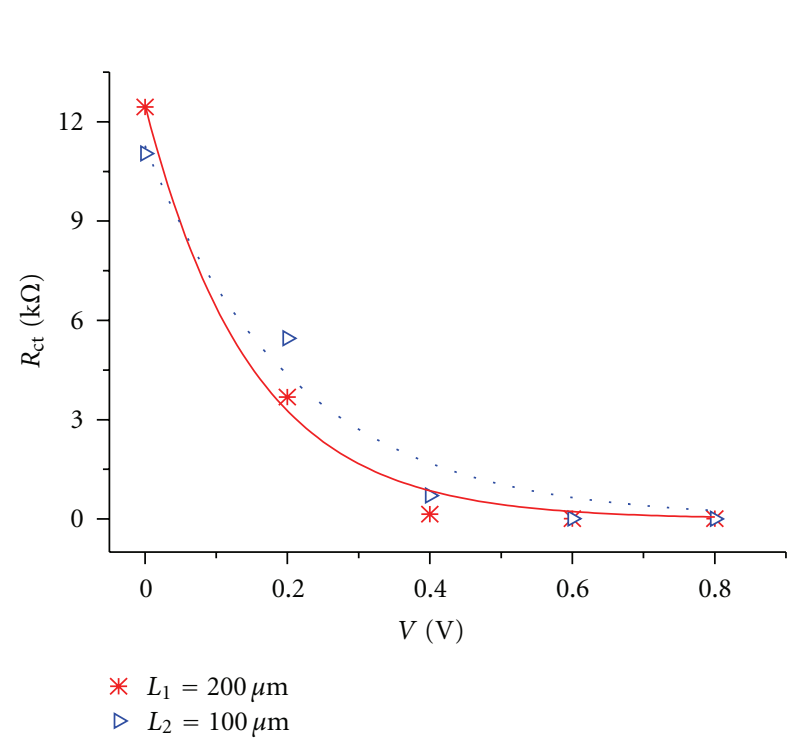

(a)
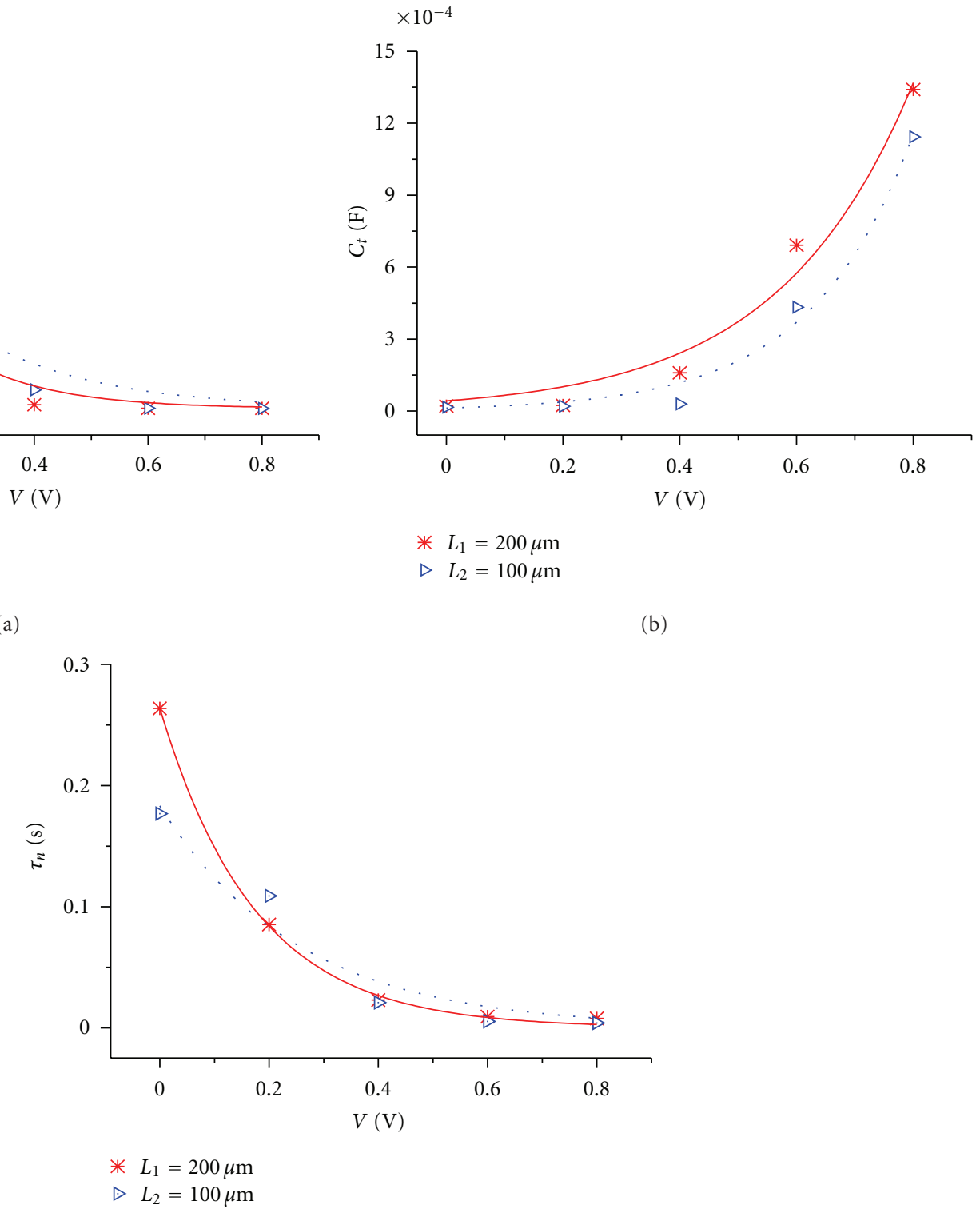

(b)

(c)

FIGURE 6: Charge transfer resistance (a), total capacitance (b), and charge lifetime (c) versus applied bias voltage for two cells with $L_{1}=$ $200 \mu \mathrm{m}$ (red stars) and $L_{2}=100 \mu \mathrm{m}$ (blue triangles), under light conditions. The continuous curves are the best fit, obtained by means of the model described in the text. $\tau_{n}=R_{\mathrm{ct}} C_{t}$ is the charge-transfer relaxation time.

the DSC and depends on the bias in an exponential manner, as it follows from the expression (12).

In Figure 7, we report the same parameters shown in Figure 6, for the DSC under dark conditions. From the best fit of Figures $7(\mathrm{a})$ and $7(\mathrm{~b})$, we find $\beta(100 \mu \mathrm{m})=0.17$, $\beta(200 \mu \mathrm{m})=0.23$ and $\alpha(100 \mu \mathrm{m})=0.25, \alpha(200 \mu \mathrm{m})=0.12$.

From the experimental data, it is possible to evaluate the diffusion coefficient $(D)$, the carrier concentration in the $\mathrm{TiO}_{2}$ layer $(n)$ and the diffusion length of the carrier in the $\mathrm{TiO}_{2}$ layer $\left(L_{n}\right)$ by means of the equations $D=d^{2} / R_{\mathrm{tr}} C_{t}$, $n=\left(k_{B} T / q^{2}\right) \cdot\left(C_{t} / S \cdot d\right)$ and $L_{n}=d\left(R_{\mathrm{ct}} / R_{\mathrm{tr}}\right)^{1 / 2}$. For our DSC of thickness $L_{1}=200 \mu \mathrm{m}$ submitted to the bias voltage of $0.6 \mathrm{~V}$, we find $D \sim 7.6 \cdot 10^{-9} \mathrm{~m}^{2} / \mathrm{s}, n \sim 1.32 \cdot 10^{23} \mathrm{~m}^{-3}$, and $L_{n} \sim 8.39 \cdot 10^{-6} \mathrm{~m}$.

\section{Conclusions}

A new microfluidic technological procedure for DSC fabrication has been proposed. Our aim is the engineering of a small laboratory cell which permits to distinguish between the contributions of the different components and technological steps. The microfluidic structure is reversibly sealed and can be interfaced with a housing system consisting of mechanical clamping, inlet/outlet ports, and interconnections to external 

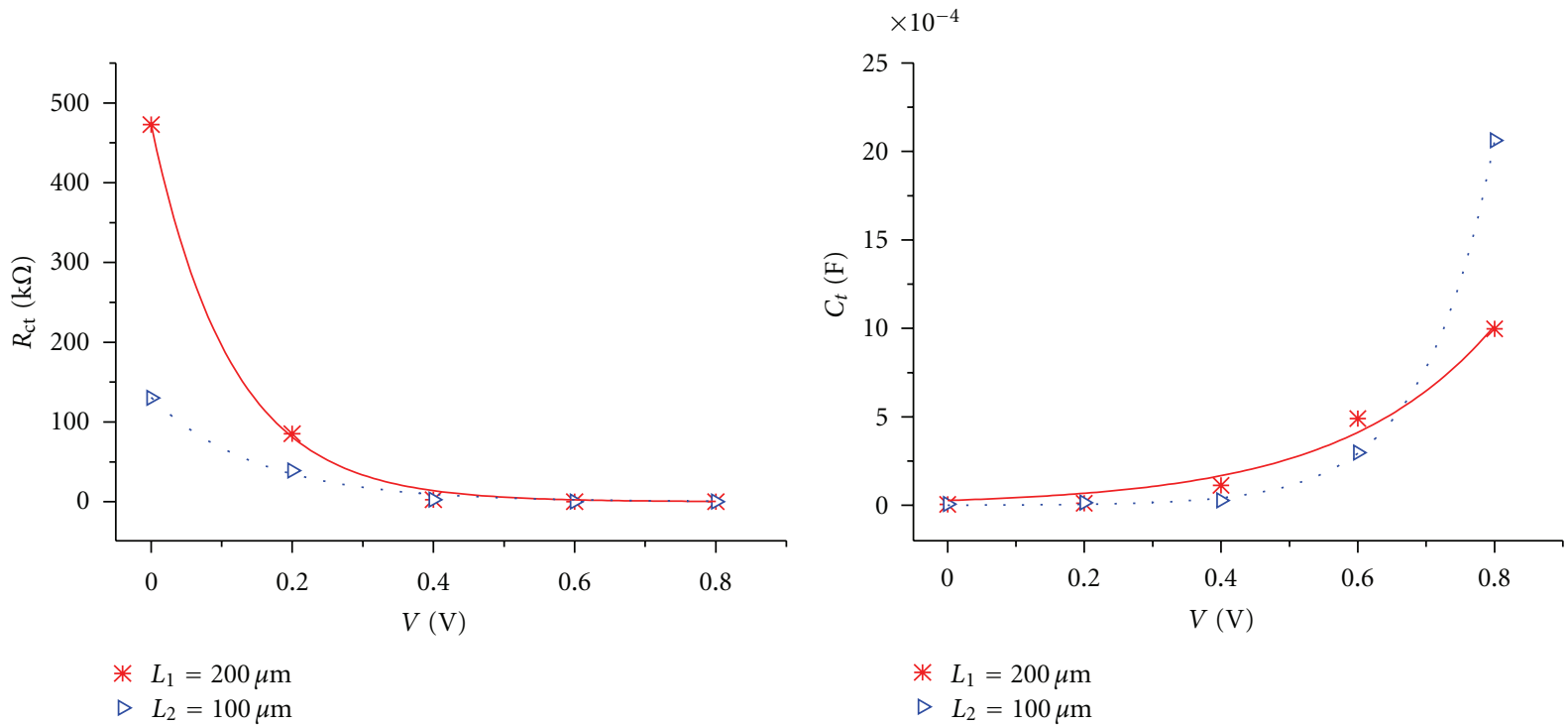

(a)

(b)

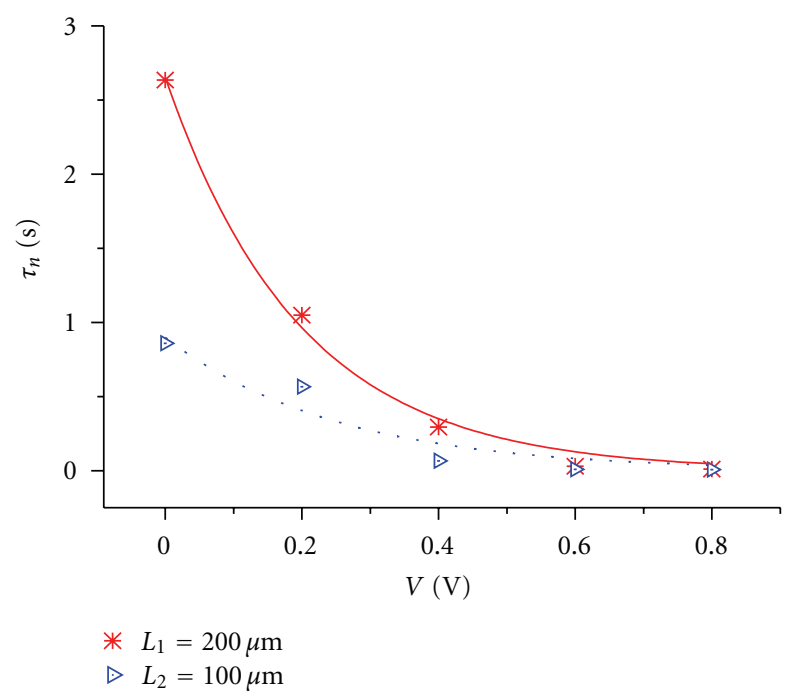

(c)

Figure 7: Charge transfer resistance (a), total capacitance (b), and charge lifetime (c) versus applied bias voltage for two cells with thicknesses $L_{1}=200 \mu \mathrm{m}$ (red stars) and $L_{2}=100 \mu \mathrm{m}$ (blue triangles), under dark conditions. The continuous curves are the best fit, obtained by means of the model described in the text. $\tau_{n}=R_{\mathrm{ct}} C_{t}$ is the charge-transfer relaxation time.

fluids handling devices. This technological approach guarantees a high degree of reproducibility, as demonstrated by a statistical analysis. Moreover, the modularity of the device enables the possibility of postprocess control and inspection.

We have investigated the electrical response of a microfluidic DSC to an external voltage of variable frequency. EIS measurements have been performed under light and dark conditions, at different bias voltages ranging from $0 \mathrm{~V}$ to $0.8 \mathrm{~V}$ and for two different thicknesses of the cells. The experimental data are analyzed by means of a transmission line model, with two transport channels, to validate already proposed models. From the spectra of the real and imaginary parts of the electric impedance of the cell, we have derived the charge-transfer resistance and total capacitance relevant to the interface porous electrode-electrolyte. The experimental data are well described by the transmission line model and the parameters characterizing the diffusion coefficient, carrier concentration, and the diffusion length of the carrier in the $\mathrm{TiO}_{2}$ layer are in agreement with the values reported in the literature.

This validation is fundamental since it shows that our microfluidic approach does not change the nature of the device, but simply adds flexibility and reliability. We demonstrate the effectiveness of the microfluidic cells as a standard modular prototype which can be used for testing different DSC components. 


\section{Acknowledgment}

The authors would like to thank D. Flore for the collaboration in cells fabrication.

\section{References}

[1] B. O’Regan and M. Grätzel, "A low-cost, high-efficiency solar cell based on dye-sensitized colloidal $\mathrm{TiO}_{2}$ films," Nature, vol. 353, no. 6346, pp. 737-740, 1991.

[2] S. Ito, T. N. Murakami, P. Comte et al., "Fabrication of thin film dye sensitized solar cells with solar to electric power conversion efficiency over 10\%," Thin Solid Films, vol. 516, no. 14, pp. 4613-4619, 2008.

[3] J. M. Kroon, N. J. Bakker, H. J. P. Smit et al., "Nanocrystalline dye-sensitized solar cells having maximum performance," Progress in Photovoltaics, vol. 15, no. 1, pp. 1-18, 2007.

[4] D. Shi, N. Pootrakulchote, R. Li et al., "New efficiency records for stable dye-sensitized solar cells with low-volatility and ionic liquid electrolytes," Journal of Physical Chemistry C, vol. 112, no. 44, pp. 17046-17050, 2008.

[5] M. Späth, P. M. Sommeling, J. A. M. Van Roosmalen et al., "Reproducible manufacturing of dye-sensitized solar cells on a semi-automated baseline," Progress in Photovoltaics, vol. 11, no. 3, pp. 207-220, 2003.

[6] M. K. Nazeeruddin, E. Baranoff, and M. Grätzel, "Dyesensitized solar cells: a brief overview," Solar Energy, vol. 85, no. 6, pp. 1172-1178, 2011.

[7] J. Halme, P. Vahermaa, K. Miettunen, and P. Lund, "Device physics of dye solar cells," Advanced Materials, vol. 22, no. 35, pp. E210-E234, 2010.

[8] G. Barbero, A. L. Alexe-Ionescu, and I. Lelidis, "Significance of small voltage in impedance spectroscopy measurements on electrolytic cells," Journal of Applied Physics, vol. 98, no. 11, Article ID 113703, pp. 1-5, 2005.

[9] G. Barbero, M. Becchi, and F. C. M. Freire, "Contribution of the electrode-electrolyte interface to the impedance of an electrolytic cell," Journal of Applied Physics, vol. 104, no. 11, Article ID 114111, 2008.

[10] G. Barbero and I. Lelidis, "Evidence of the ambipolar diffusion in the impedance spectroscopy of an electrolytic cell," Physical Review E - Statistical, Nonlinear, and Soft Matter Physics, vol. 76, no. 5, 2007.

[11] E. K. Lenzi, L. R. Evangelista, and G. Barbero, "Fractional diffusion equation and impedance spectroscopy of electrolytic cells," Journal of Physical Chemistry B, vol. 113, no. 33, pp. 11371-11374, 2009.

[12] F. Fabregat-Santiago, J. Bisquert, G. Garcia-Belmonte, G. Boschloo, and A. Hagfeldt, "Influence of electrolyte in transport and recombination in dye-sensitized solar cells studied by impedance spectroscopy," Solar Energy Materials and Solar Cells, vol. 87, no. 1-4, pp. 117-131, 2005.

[13] Q. Wang, J. E. Moser, and M. Grätzel, "Electrochemical impedance spectroscopic analysis of dye-sensitized solar cells," Journal of Physical Chemistry B, vol. 109, no. 31, pp. 1494514953, 2005.

[14] J. Bisquert, D. Cahen, G. Hodes, S. Rühle, and A. Zaban, "Physical chemical principles of photovoltaic conversion with nanoparticulate, mesoporous dye-sensitized solar cells," Journal of Physical Chemistry B, vol. 108, no. 24, pp. 8106-8118, 2004.
[15] M. Liberatore, F. Decker, L. Burtone et al., "Using EIS for diagnosis of dye-sensitized solar cells performance," Journal of Applied Electrochemistry, vol. 39, no. 11, pp. 2291-2295, 2009.

[16] F. Fabregat-Santiago, J. Bisquert, E. Palomares, S. A. Haque, and J. R. Durrant, "Impedance spectroscopy study of dyesensitized solar cells with undoped spiro-OMeTAD as hole conductor," Journal of Applied Physics, vol. 100, no. 3, Article ID 034510, 2006.

[17] S. Ito, M. K. Nazeeruddin, P. Liska et al., "Photovoltaic characterization of dye-sensitized solar cells: effect of device masking on conversion efficiency," Progress in Photovoltaics, vol. 14, no. 7, pp. 589-601, 2006.

[18] N. G. Park, J. Van De Lagemaat, and A. J. Frank, "Comparison of dye-sensitized rutile- and anatase-based TiO2 solar cells," Journal of Physical Chemistry B, vol. 104, no. 38, pp. 89898994, 2000.

[19] A. Lamberti, A. Sacco, S. Bianco et al., "Microfluidic sealing and housing system for innovative dye-sensitized solar cell architecture," Microelectronic Engineering, vol. 88, no. 8, pp. 2308-2310, 2011.

[20] J. Bisquert, "Influence of the boundaries in the impedance of porous film electrodes," Physical Chemistry Chemical Physics, vol. 2, no. 18, pp. 4185-4192, 2000.

[21] G. Barbero and A. L. Alexe-Ionescu, "Role of the diffuse layer of the ionic charge on the impedance spectroscopy of a cell of liquid," Liquid Crystals, vol. 32, no. 7, pp. 943-949, 2005.

[22] Q. Wang, S. Ito, M. Grätzel et al., "Characteristics of high efficiency dye-sensitized solar cells," Journal of Physical Chemistry B, vol. 110, no. 50, pp. 25210-25221, 2006.

[23] J. W. Ondersma and T. W. Hamann, "Impedance investigation of dye-sensitized solar cells employing outer-sphere redox shuttles," Journal of Physical Chemistry C, vol. 114, no. 1, pp. 638-645, 2010.

[24] P. W. Atkins and J. DePaula, Atkins' Physical Chemistry, Oxford University Press, Oxford, UK, 9th edition, 2009.

[25] J. Bisquert, "Theory of the impedance of electron diffusion and recombination in a thin layer," Journal of Physical Chemistry B, vol. 106, no. 2, pp. 325-333, 2002. 


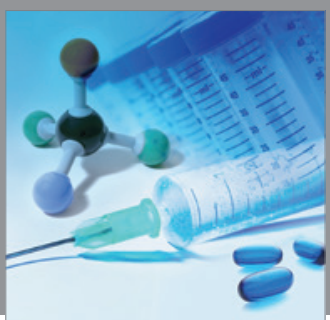

International Journal of

Medicinal Chemistry

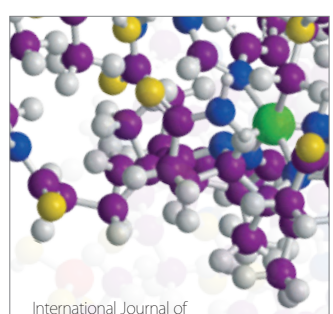

Carbohydrate Chemistry

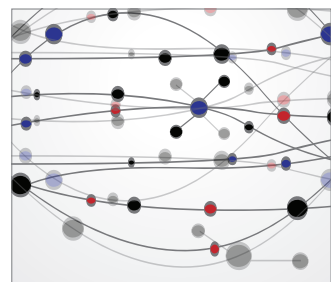

The Scientific World Journal
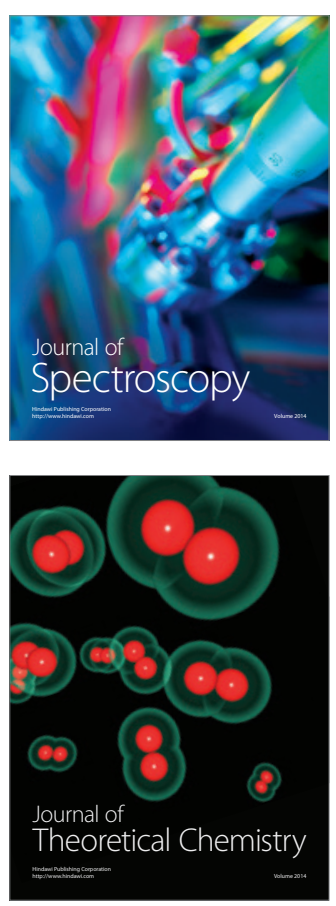
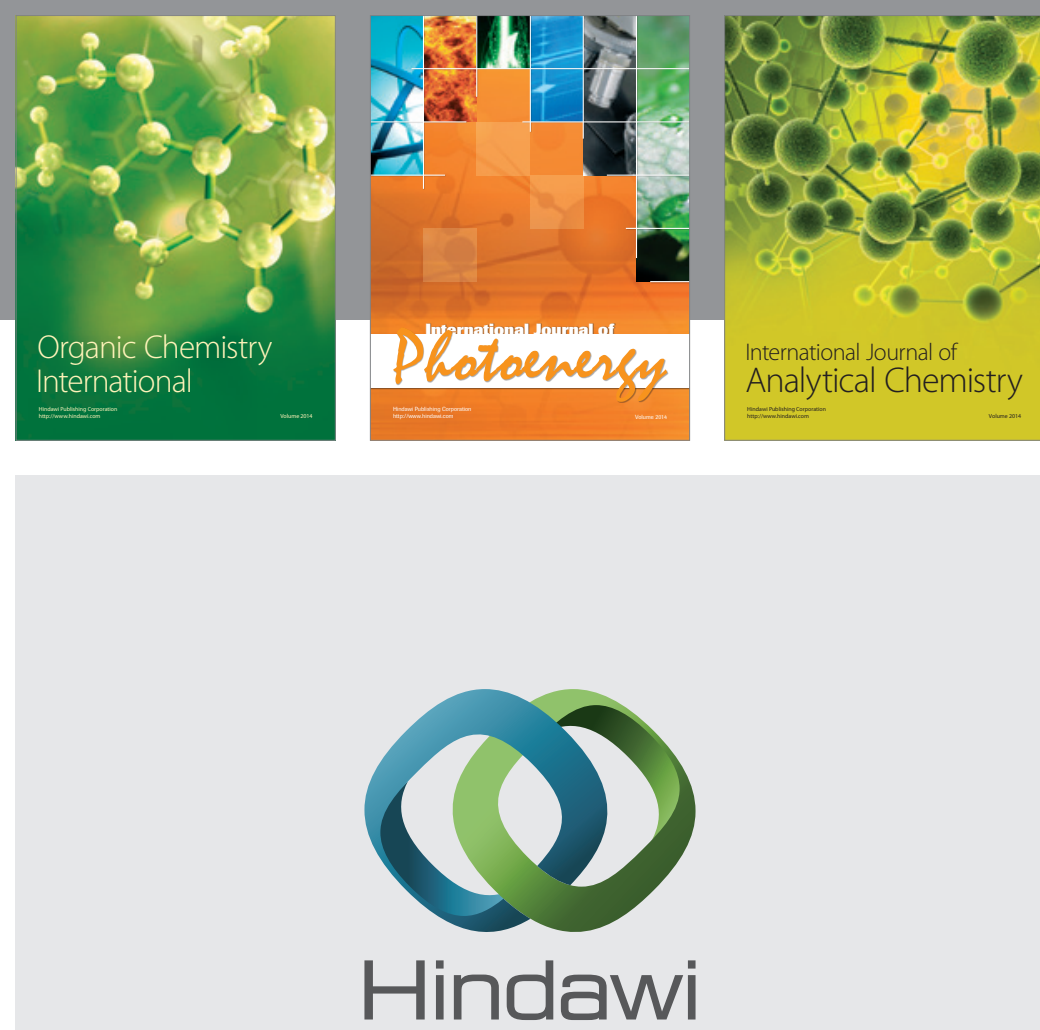

Submit your manuscripts at

http://www.hindawi.com
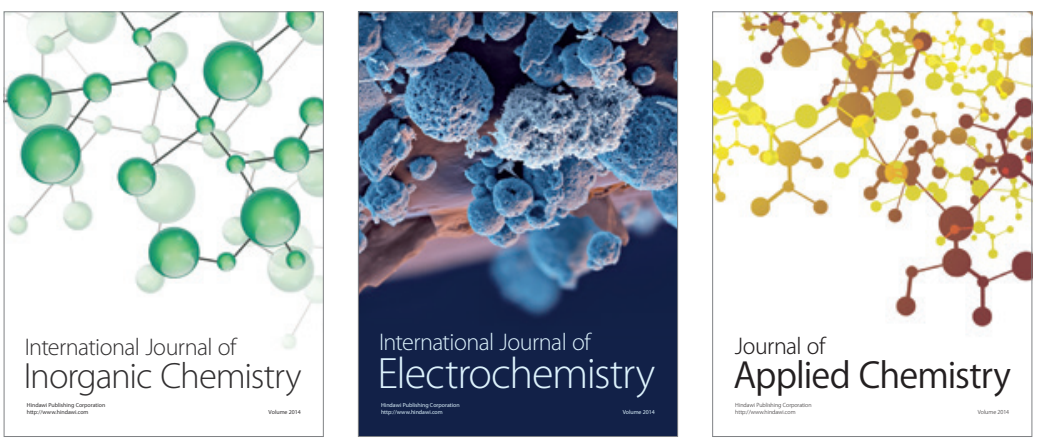

Journal of

Applied Chemistry
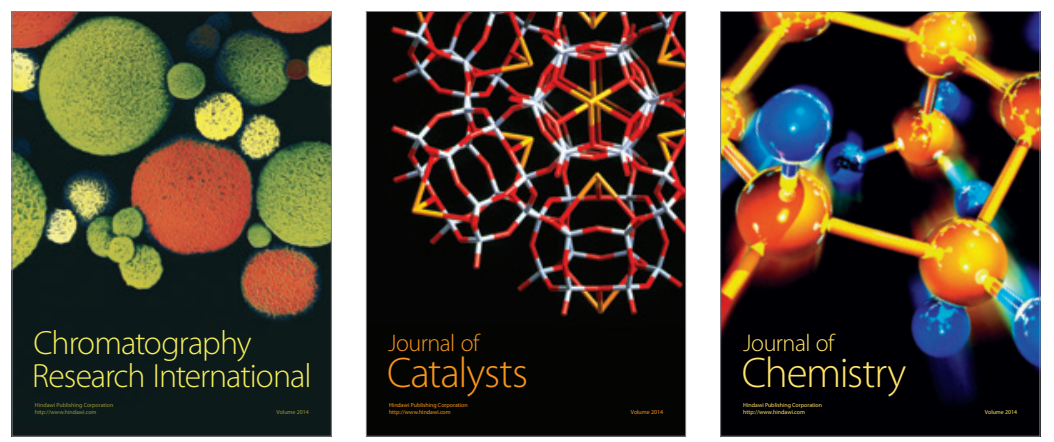
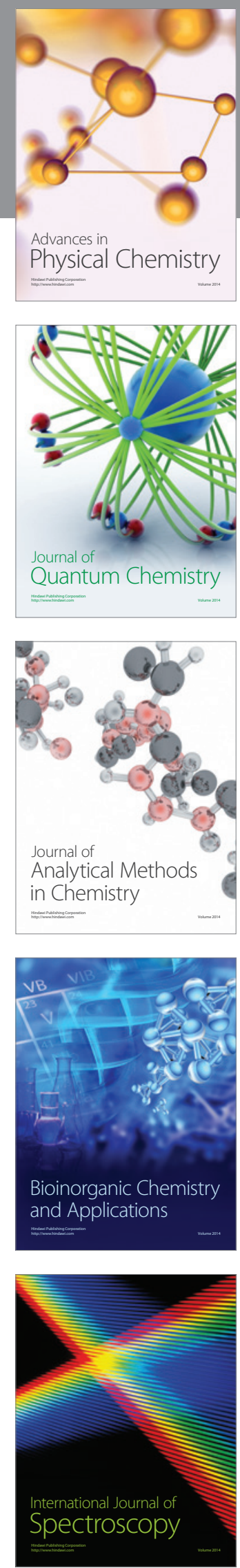